\title{
Schelmuffsky trifft Soemmerring \\ Brentanos und Görres' Bogs als teuflische Parodie
}

von Maximilian Bergengruen (Basel)

\section{Abstract}

Gegenstand meiner Untersuchung ist Joseph Görres' und Clemens Brentanos Bogs aus dem Jahre 1807. ${ }^{1}$ Mich interessiert an diesem Text das Zusammenspiel zweier seiner auf den ersten Blick vollkommen unterschiedlichen Agenten: nämlich einerseits der Physiologen Dr. Sphex, Dr. Schnauznas und Dr. Galmaliel, die Bogs' Gehirn untersuchen, und andererseits der Figuren aus Christian Reuters spätbarockem Roman Schelmuffsky (1697), auf die die Mediziner während ihrer Vivisektionen in Bogs' Gehirn stoßen. Abstrahiert man von den Figuren auf die wissens- und literarhistorischen Hintergründe der Geschichte, dann ist es mir um die Konfrontation zweier unterschiedlicher, intertextuell zu verstehender, Ebenen der Erzählung zu tun: die reiche Bilderund Vorstellungswelt eines Lügenromans aus dem ausgehenden 17. Jahrhundert und die zeitgenössische, also ziemlich genau 100 Jahre jüngere, und ungleich nüchterne Gehirnphysiologie eines Samuel Thomas Soemmering und seiner Nachfolger. Ich möchte zeigen, dass in Brentanos und Görres Bogs ein Tertium Comparationis dieser auf den ersten Blick unvereinbar scheinenden Figuren- und Text-Ebenen angeboten wird: der Teufel mit seinen fantastisch-komischen Fähigkeiten zur Parodie.

\section{Schelmuffsky ₹u Gast bei Brentano und Görres}

Ich lasse kurz die Handlung von Görres' und Brentanos Satire passieren: Der Uhrmacher Bogs bittet um Aufnahme in die, ihm durch ein Plakat bekannt gemachte, wohllöbliche Schützengesellschaft; diese hat Bedenken und knüpft die Aufnahme an eine "Bedingung": Bogs soll ein Konzert besuchen und dabei beweisen, dass er, dessen "Tollheiten über Musik" der Schützengesellschaft anscheinend schon zu Ohren gekommen sind, währeddessen nicht "zu sehr hingerissen worden" ist (Bogs 15). ${ }^{2}$

Hingerissen ist jedoch gar kein Ausdruck für das, was Bogs während der Aufführung widerfährt, ist dieser doch im Konzertsaal mit fantastischen, genauer gesagt: synästhetischen Erfahrungen konfrontiert, die die Vorstellungskraft der Schützengesellschaft bei weitem übersteigen. ${ }^{3}$ Während die Musik erklingt - es handelt sich um eine, wie sie humoristisch genannt wird, "Haidnische[ Simphonie -, spielen sich z. B. folgende Szenen vor seinem geistigen Auge ab: "Ich war auf dem Abgrund eines Meeres, alle Leute waren Fische, ich selbst eine Art Hering, ich sah mich tausendmal, da rührte Musika gewaltig, ein Waldfisch erhob sich, [...] ein Schlag mit seinem Schwanz, ein Strom, hin fuhren wir alle in seinen Rachen, da saß Jonas, der sang und lobte Gott; ich sagte ihm, daß ich ein Uhrmacher sey, und daß es eben halb sieben geschlagen" (Bogs 17).

\footnotetext{
${ }^{1}$ Ich zitiere nach: Brentano/Görres (anon.) 1807 (Sigle 'Bogs') direkt im Haupttext.

2 Zu den hier ausgeklammerten musiktheoretischen Bezügen im Bogs, vgl. Lubkoll 1995, $163 \mathrm{ff}$.

3 Zur - nicht gehirnphysiologisch gedachten - Synästhesie im Bogs, vgl. Utz 1990, 177ff. (Romantische Synästhesie allgemein); 247ff. (zum Bogs).
} 
In den Pausen der von Bogs so vielsinnig wahrgenommenen Symphonie, und zwar durchaus in deren "Folge" (Bogs 19), sind es nun weniger die Figuren des Alten Testaments denn Reuters Schellmufsky, die als Ideen- und Figurengeber von Bogs' Fantasmagorien figurieren: Der Uhrmacher nimmt schon bald hinter sich verschwommen eine Person wahr, die er mit dem "seelige[n] Schelmufski" (Bogs 19) identifizieren zu können glaubt.

Selig ist Schelmuffsky deswegen, weil er bei Brentano/Görres eine "elendige Ermordung durch den holländischen Generalstaaten" erleiden musste. Hier tritt dieser todgeweihte Schelmuffsky jedoch an einer früheren Stelle seines Lebens auf: "Mir schauderte die Haut, denn sein Mörder selbst stand in bester Freundschaft neben ihm" (Bogs 19). Daneben zwei weitere Figuren von Reuters Roman (und engste Begleiter des Protagonisten): "die Dame Charmante [...] und der Herr Bruder Graf" (Bogs 19f.).

Doch Bogs ist der Einzige, der diese vier literarischen Figuren zu sehen in der Lage ist; sein Nachbar, anscheinend ein Mitglied der Schützengesellschaft, behauptet, der angebliche Schelmuffsky sei "ein Bilderhändler vom Comersee aus Tremezzo, der nicht an den Takt glaube, und sich studierenshalber hier aufhalte über die Leute, der andre aber", gemeint ist wahrscheinlich der Herr Bruder Graf, "sey ihm unbekannt und esse gern weiße Bohnen, doch lieber noch grüne Erbsen" (Bogs 20).

Diese grobe Lüge bekommt dem auskunftsfreudigen Herrn allerdings überhaupt nicht. Als dieser nämlich dem Uhrmacher unter einem fadenscheinigen Vorwand eine seiner Uhren klauen möchte, meldet sich Schelmuffsky von hinten zu Wort: "So haben wir nicht gewettet, hier gehts nicht, wie bei der Äpfelfrau". Und auch musikalisch erweist sich der selige Schelmuffsky als durchaus in der Lage, das Gehörte einzuordnen: "o Sapperment! dergleichen Blasen hab ich noch nicht gehört, das war, der Tebel hol mir, grade wie zu Akra, als ich beim großen Mogol oben an zu Tische saß, die große Mogolin saß zur Rechten, und drückte mir immer die Fäuste und gab Freiens bei mir vor" (Bogs 23).

Beim weiteren Referat dieser - in Reuters Roman im Übrigen zentralen - Passage, dem Besuch Schelmuffskys beim Mogol (auf ihn wird zurückzukommen sein), ist sich Bogs nicht mehr sicher, ob der barocke Lügenerzähler selbst oder sein Nachbar spricht; schließlich könnte dieser Schelmuffskys Formulierungen "abgehorcht" bzw. die wichtigsten Passagen des Romans "auswendig gelernt" haben. Doch genau lässt sich das nicht mehr eruieren: "Es war ein rechtes Elend, immer wenn ich mich zu dem verblichenen Freunde umwenden wollte, ging die Musik wieder an, und faßte mich der Wahnsinn" (Bogs 26; 29).

Der 'verblichene Freund': Da Totgesagte bekanntlich länger leben, tritt Schelmuffsky noch ein weiteres Mal auf. Mittlerweile hat die Schützengesellschaft - der Bericht des Uhrmachers hat ihre Befürchtungen eher bestätigt, denn verringert - ein medizinisches Kollegium um eine Analyse von Bogs' Geistes-, genauer: Gehirnzustand gebeten. Die Mediziner erwägen zuerst eine Trepanation, entschließen sich jedoch dann für eine wesentliche weniger aufwändige Visitation der vorderen Hirnhöhle durch die - zum Glück hohlen - Geruchsnerven. Und nun treffen auch sie auf Schelmuffsky: "In dem Augenblicke sahen wir einen Schellenschlitten vor dem Thore der dritten Stirnhöhle gewöhnlich Anus genannt, anfahren, auf Befragen des Torschreibers wer die Passagiere wären, schrie's zum Fuhrwerk heraus: Herr von Schelmufski, samt Herr Bruder Graf, auf weiteres Befragen um Ziel und Zweck der Reise der hohen Herrschaft, hieß es, wir wollen, der Tebel hol mir, flugs die berühmte Wasserleitung des Herrn Sylvius besehen, und der Dame 
Charmante einige Liebesäpfel vom Lebensbaume abpflücken. Passiren, und nun fuhr der Schlitten klingelnd in den Pausilipp hinein" (Bogs 46f.).

Die "Wasserleitung des Herrn Sylvius" ist der Aquaeductus Sylvii, der von Franciscus Sylvius entdeckte und nach ihm benannte Verbindungskanal zwischen dem (den Medizinern) einsehbaren dritten und dem (ihnen verborgenen) vierten Hirnventrikel, der Pausilipp eigentlich ein Gebirgszug bei Neapel. In diesem Falle ist wahrscheinlich die im 18. Jahrhundert des Öfteren besungene Grotte im Pausilipp gemeint, die hier metaphorisch mit der vierten Hirnhöhle gleichgesetzt wird. In diese Hirngrotte fahren also Schelmuffsky und sein Bruder Graf auf Nimmerwiedersehen. Grund genug, anlässlich ihres Verschwindens endlich ihres Herkommens zu gedenken.

\section{Der Teufel als stiller Protagonist des Schelmuffsky}

Die Schelmuffsky-Geschichten im Bogs sind weder Zitate noch Paraphrasen des von Brentano und Grimm so verehrten Schelmuffsky (dessen Autor, Christian Reuter, ihnen damals noch unbekannt war). Sie sind auch nicht der in der B-Ausgabe angekündigte, aber (von einer anonymen Fortsetzung aus dem Jahre 1792 einmal abgesehen) ${ }^{4}$ nie veröffentlichte "dritte[ Theil[" von Schelmuffskys "gefährliche[r] Reisebescheibung". ${ }^{5}$ Es handelt sich eher um Umdichtungen und Variationen, die im 'Schelmuffsky-Kultus', ${ }^{6}$ den die Gebrüder Grimm, Arnim und Brentano in Gesprächen und Briefen auf der Grundlage des Barockromans auch an anderer Stelle betreiben, häufig vorkommen. In diesem Falle könnte man von Anpassungen der Weltreise, die Schelmuffsky in seinem Lügenroman unternimmt, zur Gehirnreise, die Dr. Sphex in Bogs Gehirn macht, sprechen: Von einer Reise "zu Wasser und Lande", wie es im Titel des Schelmuffsky heißt, zu einer Reise zu den festen Teilen der Gehirnhöhlen und zur Gehirnhöhlenflüssigkeit. Man denke z. B. an die Schlittenfahrt des Bruder Grafen und Schelmuffsky in die vierte Gehirnhöhle, die im barocken Original von Schelmerode nach Hamburg führt.

Manchmal sind die Varianten noch freihändiger: Der angebliche Mörder Schelmuffskys z. B., von dem Bogs währende des Konzertes spricht, findet bei Reuter keine Erwähnung. Im Original wird der Protagonist des Lügenromans nämlich nicht von einem holländischen Generalstaaten im Zorn ermordet, sondern er bekommt lediglich von einem Stockholmer Nebenbuhler, weil er ihm im Handstreich die Frau weggeschnappt hat, eine "Presche[n]", also eine Ohrfeige, von der er sich jedoch recht schnell erholt (Schelmuffsky 46). Vielleicht spielen Brentano und Görres aber auch auf die Episode an, da sich eine "Staadens-Tochter" (Schelmuffsky 73f.) in Schelmuffsky verliebt. Aber auch hier ist die Abweichung überdeutlich: Bei Reuter weiß der Vater die Verbindung ohne Mord, d. h. durch ein einfaches Untersagen des Umgangs, zu verhindern. Es gibt jedoch neben dem hier skizzierten Verhältnis von Original und Variation noch einen zweiten Verbindungspunkt zwischen dem Bogs und dem Schelmuffsky - und das ist die Rolle, die

\footnotetext{
4 Vgl. hierzu Polenz: Einleitung. In: Reuter 21956, XVII.

${ }^{5}$ Reuter 1997, 190. Diese Edition folgt den Originalausgaben: Reuter (anon.) 1696 (B-Auflage erster Teil) und Reuter (anon.) 1697 (A-Auflage, zweiter Teil). Brentano und Görres lag höchstwahrscheinlich Reuter (anon.) 1750 vor, nach der ich unter der Sigle 'Schelmuffsky' direkt im Haupttext zitiere.

${ }^{6}$ Vgl. hierzu Rölleke 2004, 205ff. mit Bezug auf Deneke 2005, 305-308. Ähnlich Rölleke 2005.
} 
der Teufel in beiden Texten spielt. Beim Schelmuffsky kann man diesbezüglich, wie ich im Folgenden ausführen möchte, von einer Art stillen Protagonisten sprechen,

Es sind zwei, dafür jedoch unendlich oft wiederholte, Redensarten, die die Erzählweise von Christian Reuters Roman Schelmuffsky - und damit auch seines gleichnamigen Protagonisten auszeichnen: "der Tebel hohlmer" und "Sapperment". Die beiden Begriffe bezeichnen sehr genau die Spanne, innerhalb deren sich ein Mensch im christlichen (und dem Hexenwahn verfallenen) Zeitalter des Barock bewegen kann. Der Teufel holt einen, genauer gesagt: einen Mann, bekanntlich nicht ohne Vorwarnung: Meist wurde ein Vertrag, ein Teufelsbund, abgeschlossen, dessen teuflische Rechte darin bestehen, dass der Teufel die Seele des Menschen zu einem gewissen Zeitpunkt erhält. Ganz im Gegenteil dazu das Wort "Sapperment", eine deutsche Verquatschung des Wortes 'Sakrament'. Auf einen Nenner gebracht: Schelmuffsky bewegt sich in seinen Redensarten zwischen nichts weniger als Himmel und Hölle.

Doch die beiden Begriffe sind noch enger miteinander verbunden. Der männliche Teufelsbund bzw. die weibliche Vereinigung mit dem Teufel figurieren nämlich, wie ich hier nur andeutungsweise ausführen kann, in der Frühen Neuzeit als inverse Form des Sakraments; im Falle des Mannes: des Taufbundes; im Falle der Frau: des Ehe-Versprechens. So wie der Mensch bei der Taufe nach Luther einen "bund["mit Gott eingeht, innerhalb dessen er verspricht, "die sund zu tödten" und im "glauben" zu leben, ${ }^{7}$ Gott im Gegenzug dem Menschen, dass des Menschen Peccatum originale "auß getilget wirt teglich mehr und mehr biß yn den todt", ${ }^{8}$ so schließen auch Mann und Teufel einen fast wortgleichen Bund, nur in diesem Falle unter inversen Vorzeichen. Der Mensch verspricht dem Teufel, seinen zuvor geschlossen "bund" mit Gott zu invertieren, also der abgeschworenen Tat-Sünde wieder zuzusprechen, der Teufel dem Menschen, ebenfalls den ursprünglichen Vertrag invertierend, den Menschen statt dem himmlischen dem irdischen Heil entgegen zuführen (mit dem geheimen Zusatzabkommen, dass das Peccatum originale so erhalten bleibt und der Teufel dadurch Gewalt über den Menschen erlangt). Man könnte sagen, dass die Umschreibung des Taufbundes in den "falsche[n] begriegliche[n] [...] Bundt / welchen der Teuffel auß seiner arglistigkeit / durch viel vnnd mancherley betrug vnnd verblendung / deß arbeitseligen Menschen anbrittlet", ${ }^{9}$ als die Urform aller Parodie zu lesen ist.

Der Teufel als Parodist des göttlichen Wortes: Das damit implizierte leichte und komische Element des Teufels scheint im Schelmuffsky seine volle Entfaltung erreicht zu haben, sind doch die beiden Ausdrücke, die auf den Teufelsbund und seine parodische Invertierung des Sakraments hinweisen, also "Sapperment" und "Der Tebel hohlmer", erstens als sprachlich verschliffen und zweitens als Füllworte, ja als unbewusstes Sprechverhalten markiert. Der Teufel und seine begrifflichen Invertierungen scheinen also ihre metaphysische Schwere verloren und stattdessen eine, gleichsam mitlaufende, Leichtigkeit erlangt zu haben.

Dieser Umstand hat eine literarische Vorgeschichte: Den Anfang der hier thematisierten Wendung des Teufels ins Spielerische macht der diskursiv, leider nicht in der Realität, erfolgreichste Gegner der Hexenverfolgung Johann Weyer. Dieser argumentiert in seinem

\footnotetext{
${ }^{7}$ Luther: Taufsermon. In: Luther 1883ff., Bd. II, 730, 733.

${ }^{8}$ Ebd., 731.

${ }^{9}$ Weyer 1586, $1499_{2}$ (vollständige, aber nicht autorisierte Fassung).
} 
Hauptwerk, De praestigiis demonum von 1563, dass dem Teufel keine reale Einflussnahme auf die Natur und den Menschen zu Gebote stünde. Die einzige mögliche Form von Manipulationen, die ihm bliebe, sei eine psychische, nämlich in die "Fantasey" des Menschen ${ }^{10}$ eine "falsche einbildung" ${ }^{11}$ bzw. ein Trugbild, also die schon im Titel des Werkes genannten "Praestigi[ae]", zu installieren. Leicht einzusehen, dass hier die Literatur aufhorchen musste, stehen ihr doch ebenfalls keine realen Veränderungen von Natur und Mensch, sondern nur die fantastische Antäuschung von Sachverhalten zu Verfügung.

Dass eine solcher Transfer vom Teufel auf den Menschen möglich ist, kann man schon bei Hans Michael Moscherosch erfahren, der im ersten der Gesichte Philanders von Sittewald behauptet hatte: "jeder Mänsch ist fast des andern Teuffel offt mehr als der Teuffel selbsten, Homo homini lupus. Homo homini Diabolus". ${ }^{12}$ Daraus schließt wiederum Hans Jacob Christoffel von Grimmelshausen, dass "das gemeine Sprichwort auf Erden nit durchaus erlogen / wann man nemlich spricht: Es seye je ein Mensch des andern Teuffel". ${ }^{13}$ "Homo homini Diabolus" - das heißt soviel wie: Auch der Mensch beherrscht die teuflische Fähigkeit, die Fantasie anderer so zu manipulieren, dass sie ihre vorgegaukelten Vorstellungen für Erfahrung halten (vielleicht sogar besser als der Teufel selbst).

Dies zumal, da Johann Weyer seinem Argument, dass der Teufel zu keiner realen Veränderung der Schöpfung fähig sei, also lediglich auf die Möglichkeit zu Praestigiae beschränkt sei, präzisierend hinzugefügt hatte, dass dieser seine zu introduzierenden Fantasiebilder nicht frei erfinde, sondern in diesem Zusammenspiel ein "apffenspiel" des "Göttlichen wort[es]" und ein "nach äffen" der "wercke[ gottes" aufführe, also die göttlichen Vorgaben aufnehme, jedoch durch eine "zusetzung" - er bezieht sich dabei auf die zu seiner Zeit gängige Etymologie des Namens Leviathan - so manipuliere, dass sie auf das Gegenteil des göttlichen Schöpfungsplanes hinausliefen. ${ }^{14}$ Der Teufel ist, so präzisiert Georg Philipp Harsdörffer diesen Gedanken in seinem Schau-Plat乏jämmerlicher Mordg-Geschichte, ein "Aff deß Allmächtigen" und wiederholt alle göttlichen Werke und Worte "in verkehrtem Verstand". ${ }^{15}$ Nachahmung, Differenz-Markierung und Invertierung der ursprünglichen Intention - damit sind (wenn man die pejorativen Teufelszuschreibungen vom Kopf auf die Fuße stellt) drei von vier basalen Merkmale einer Parodie gegeben. ${ }^{16}$

Und auch das vierte Merkmal, nämlich das Abzielen auf das Lachen, fehlt in der Tradition der literarischen Adaption des Teuflischen nicht. Simplicius Simplicissimus, der Protagonist von Grimmelshausens gleichnamigen Roman, reflektiert nämlich die geschilderte menschliche Aneignung teuflisch-parodischer Techniken, wenn er seiner Umwelt - sozusagen in einer Art von Meta-Praestigiae bzw. Meta-Nachäffung - dadurch in Angst und Schrecken versetzt, dass er sich wie ein Teufel verkleidet, also den Meister der Nachahmung und Parodie noch einmal nachahmt

\footnotetext{
10 Weyer 1967, 44 r. (eigene Übersetzung, daher autorisiert, aber nicht vollständig). Vgl. hierzu ausführlich Vf. 2007a, $235 \mathrm{ff}$.

11 Weyer 1967, 43 r.

12 Moscherosch 1964, 12; 28.

${ }^{13}$ Grimmelshausen: Verkehrte Welt. In: Grimmelshausen 1989-1997, Bd. II, 421. Herv. M. B.

${ }^{14}$ Weyer 1967, 7 v; 12 r; 52 v.

15 Harsdörffer 1974, 436.

${ }^{16}$ Ich beziehe mich hier wie im Folgenden auf Bachtins Theorie der Parodie. Vgl. hierzu Bachtin 1995, 52ff.; 345ff.; 413ff., sowie Bachtin 1979, 192ff. Vgl. auch meine, auf Bachtin aufbauenden, Ausführungen in Vf. 2003 und Vf. 2005.
} 
und sein, im Übrigen durchaus erfolgreiches, Vorgehen, statt in seinen eigenen materiellen Vorteil zu überführen, der Eigendynamik eines karnevalesken Humor anheimstellt. Nachdem Grimmelshausens Held - ich greife die erste der Imitatio-Diaboli-Passagen des Romans heraus die Schnaphanen in Gestalt des Teufels in die Flucht schlagen konnte, "lachte" er "unterdessen so schröcklich / daß es im gantzen Wald erschallete". ${ }^{17}$

Die Vortäuschung von Sachverhalten in der Fantasie seines Gegenüber als komische Parodie praktischer und diskursiver Versatzstücke - genau diese beiden depotenziert-teuflischen Attribute lassen sich auch auf Schelmuffsky anwenden. Nimmt man die Beteuerungen des Erzählers Ernst, er "habe [...] Zeitlebens kein Geprahle oder auf Auffschneidens [...] hergemacht", in seinem Buch sei also "nicht ein eintziges Wort erlogen", so kann man sich des Verdachtes nicht erwehren, dass es bei den curiösen Ereignissen, deren in der "Reise-Beschreibung" gedacht wird (Schelmuffsky )( 5 v.-6 r.), nicht mit rechten Dingen zugeht. Insbesondere im ersten Teil des Romans ist der Protagonist mit außergewöhnlichen Gaben gesegnet: Ohne jemals Unterricht erteilt bekommen zu haben, ficht Schelmuffsky wie ein Meister; nur einer immensen Überzahl von "100" Seeräubern - und auch da nur in einer Art von Remis (er hat "weder Hieb noch Stich davon getragen"; Schelmuffsky 96) - muss er sich ergeben. Bei jeder Tanzveranstaltung springt er, der niemals das Tanzen erlernt hat, höher und graziler als alle Tanzmeister zusammen, was ihm insbesondere die Bewunderung der Damenwelt einbringt: "Sapperment! wie sahen sie mir alle auf die Beine, weil ich sie so artig setzen kunte!"18 (Schelmuffsky 70)

Überhaupt die Damenwelt. Diese kann Schelmuffsky - auch das scheint nicht mit natürlichen Dingen vor sich zu gehen - beinahe geschlossen an sich binden zu können, insbesondere wenn er die Geschichte von der Ratte erzählt, die seiner Mutter ein "abscheulich groß Loch" (Schelmuffsky 111) ins "gantz neu seiden Kleid" gefressen hat und sich dann, um nicht "todt geschlagen" zu werden, "in ein Loch" in der Wand verkriecht, was wiederum Schelmuffsky dazu bewegt, ebenfalls durch ein "Loch [...] sporenstreichs in die Welt gekrochen" zu kommen (Schelmuffsky a1 r.). ${ }^{19}$

Nimmt der Erzähler und Held von Reuters Roman in dieser unverhohlen ödipalen Geschichte schnell die Rolle seines - nur durch die Chiffre der Ratte überhaupt erkennbaren - Vaters ein, so animiert er anscheinend zugleich die zuhörenden Frauen, die auf Reisen vakante Rolle der Mutter zu besetzen: "Wegen der Begebenheit von der Ratte" hat sich nicht nur die Charmante in Hamburg in Schelmuffsky "verliebet" (Schelmuffsky 17), sondern auch "Fräulein Lisette" sowie eines "vornehmen Nobels Tochter" mit Namen "Damigen" in Stockholm (Schelmuffsky 44), die "Staadens-Tochter" in Amsterdam (Schelmuffsky 70) sowie die "Lords-Töchter" (und zwar "alle mit einander") in London (Schelmuffsky 90). Und alle diese Damen sind auch untröstlich, wenn sich Schelmuffsky für die Erfüllung ihres spontanen Heiratswunsches ein wenig Bedenkzeit lässt,

\footnotetext{
${ }^{17}$ Grimmelhausen: Simplicissimus. In: Grimmelshausen 1989-1997, Bd. I.1, 173.

18 Zur Stereotypie dieser Erzählmuster, vgl. Müller 1994, $7 \mathrm{f}$.

19 Bisher hob die Forschung vor allem auf die komische narrative Nobilitierung des Helden durch die RattenGeschichte (als Parodie des Prahlens mit guter Abstammung) ab. So z. B. Tatlock 1992, 304f. Fechner 1982, 8, und Geulen 1972, 484, vermuten eine teuflische Konnotation, müssen sich aber den Vorwurf gefallen lassen, dass dieser Hinweis, wenn er sich nicht im Roman erhärten lässt (es handelt sich ja um die Exposition) ein "blindes Motiv" darstellte, also für die Analyse des Romans wenig hilfreich sei (Müller 1994, 6, FN). Nachzuweisen, dass es sich bei der teuflischen Geburtsszene nicht um ein blindes Motiv, sondern um die Thematisierung des Strukturprinzips des Romans handelt, ist das Anliegen von Vf. 2007b.
} 
was im Übrigen immer dazu führt, dass die Ehe nicht zustande kommt. Ein Kämpfer, ein Tänzer und vor allem ein Frauenverführer ohne Negativ-Saldo; kein Zweifel also, dass hier der Teufel im Spiel ist.

Glaubt man Schelmuffsky aber nicht, geht man also wie sein kleiner Vetter (der im übrigen während der Reisen seine ödipale Stelle bei der Mutter eingenommen hat) davon aus, dass "Schelmuffsky nicht weiter als eine halbe Meile von seiner Geburts-Stadt kommen wäre" und im Wirtshaus aller Geschichten "erstuncken und erlogen" habe (Schelmuffsky 113f.), dann ist der Teufel, entsprechend der oben skizzierten Umbesetzung, an eine andere Stelle der Narration getreten. Der Verdacht liegt nämlich nahe, dass der in der Erzählung ausgesparte Vater des lügenden Schelmuffsky ${ }^{20}$ der Vater aller Lügen, also der Teufel (gemäß Io 1, 44) selbst, ist. Die Tatsache, dass dieser Vater - von ein paar verräterischen Spuren wie einer abgegriffenen Redewendung einmal abgesehen - weitgehend von der Textoberfläche getilgt wurde, legt weiterhin nahe, dass es nicht nur bei der biologischen Mutter, sondern auch bei der, wie es im Titel der B-Ausgabe heißt, "Hochteutsche[n] Frau Mutter Sprache"21 zu einem Konkurrenzkampf zwischen Schelmuffsky und seinem teuflischen Vater kam, den Schelmuffsky wiederum für sich entscheiden (und damit den lästigen Konkurrenten verjagen) konnte. Statt also seinen Vater, den Teufel, zu thematisieren, wie er mithilfe seiner Fantasie Sachverhalte vortäuscht, übt Schelmuffsky diese Fähigkeit, über die er qua Erbgut ebenfalls verfügt, mit seiner Mutter, der hochdeutschen Sprache, allein aus - nämlich in der Narration seines Textes, der sich bei genauer Lektüre als eines erweist: als perennierende Lügengeschichte.

Diese Verschiebung des Teuflischen von der Textoberfläche in deren Struktur lässt sich auch an der zweiten diabolischen Eigenschaft, nämlich der Fähigkeit zur Parodie, nachweisen. Der Schelmuffsky-Roman ist bekanntlich eine Reisebeschreibung. Und genau wie andere Reisebeschreibungen der Zeit plagiiert auch Reuter bzw. der Erzähler Schelmuffsky andere, zuvor veröffentlichte Texte dieses Genres. Im Falle der bereits oben angesprochenen Episode beim Großen Mogol in Indien handelt es sich um die von Adam Olearius herausgegebene Morgenländische Reyse-Beschreibung Johan Albrecht von Mandelslos, Schleswig 1658, und Jürgen Andersens Orientalische Reise-Beschreibung, Schleswig 1669; letztere zusammen abgedruckt mit der Ost-Indischen Reise Volquard Iversens. ${ }^{22}$

Allerdings bezieht sich Reuters bzw. Schelmuffskys Plagiat nicht nur auf die Fakten - also die Informationen über das Reich des großen Mogols -, sondern auch die Techniken und Strukturen seiner Vorgängertexte. Er stellt durch dieses metareflexive Verfahren aus, wie Mandelslo, Andersen und Iversen voneinander und anderen Vorgängertexten abschreiben und wie sie die

\footnotetext{
${ }^{20}$ Die Behauptung, dass Schelmuffsky eine Figur der Bramarbas-Tradition darstellt, wurde zuerst von König 1945 , 7f. u. 62ff., vertreten (dagegen stellt sich Hecht 1966, 34f.). Die Einordnung in die Pikaro-Tradition (Schelmuffsky als falscher Pikaro, der die Kluft zum angestrebten Galanthomme-Ideal verdecken möchte) leistet Grimm 1992 (ihm folgt Bauer 1994, 139; in eine ähnliche Richtung wies schon die Argumentation von Hecht 1966, 33ff, sowie, historisch breit abgesichert [insbesondere durch den Rekurs auf Nicolas Faret], Fechner 1982, 23). In eine ganz andere Richtung verweist die Arbeit von Elsaghe 1991, der die Reisen Schelmuffskys als komisches Superstrat der Odyssee liest.

${ }^{21}$ Reuter 1997, 3. Auf die Analogie von Mutter und "Hochdeutsche Frau Mutter Sprache" macht Kaminski 2004, 256f., aufmerksam.

22 Vgl. hierzu Fechner 1982, 13f. Fechner überwindet die traditionelle Forschungsmeinung, dass Schelmuffskyse Reisebeschreibung sich allein über die für den Pikaro-Roman typische Reise verstehen lasse (so z. B. Geulen 1972, 486f.).
} 
heimatlichen politischen Verhältnisse und Topographien in die Fremde projizieren und dabei (natürlich) das Eine oder Andere hinzuerfinden. Nirgends deutlicher zu sehen als bei

Schelmuffskys Besuch beim Großmogul, dessen Reich, Hof und Zeremoniell geradezu fatal (also etwas zu auffällig) an deutsche Verhältnisse der Zeit erinnern, was gleich zu Anfang der Episode (also bei der Ankunft in Indien) deutlich herausgestrichen wird: "Ich erkundigte mich nun gleich, wo der grosse Mogol residirete; Erstlich fragte ich einen kleinen Jungen, welcher auf derselben Pfingst-Wiese, wo wir ausgestiegen waren, in einem grünen Käpgen dort herum lief und die Jungen Gänßgen hütete" (Schelmuffsky 76).

Die Lügen des Sohns des Vaters aller Lügen sind also, wie vor allem der zweite, selbstreflexive Teil des Romans andeutet, ${ }^{23}$ ein Paradigma freier Fantasie - oder zumindest weit gehend freier Fantasie. Denn wie es sich für einen teuflischen Deszendenten gehört, arbeitet der Erzähler bei näherem Hinsehen im Rahmen eines parodischen Ordnungsschemas; d. h. seine Lügen sind nach Art des bestehenden diskursiven Materials organisiert, dann aber mit fantastischen Spürsinn neu geordnet und mit einem leviathanischen Zusatz versehen, der die Implikationen des VorgängerTextes, nämlich den Glauben zu erwecken, man habe alle diese Länder gesehen und alle Erlebnisse wirklich erlebt, ins Gegenteil verkehrt.

\section{Gehimphysiologie}

Zurück zu Brentanos und Görres' Bogs: Auch hier fungiert der Teufel, wenn nicht als stiller Protagonist, denn als Enthymem der Logik des gesamten Textes. Schelmuffsky und der Bruder Graf haben mit ihrer Durchfahrt durch die "Wasserleitung des Herrn Sylvius" in die vierte Hirngrotte die Mediziner nämlich auf eine Idee gebracht. Schon zu Beginn ihrer Untersuchungen konnten sie sich in Bezug auf Bogs, sein Verhalten und vor allem sein Gehirn "des Verdachts von Zauberei und bösen Künsten", ja eines Gefühls von "Nähe des Gott sey bei uns" - also des Teufels - nicht erwehren, zumal sie ihre anfängliche Vermutung in "in der Folge" der Untersuchungen "bestätigt fanden" (Bogs 45f.). Die Frage ist nur: Wo genau im Gehirn des Bogs haust dieser, von ihnen gesuchte, ja gejagte und zu vertreibende Gottseibeiuns?

Das erste Verdachtsmoment erwächst aus einer fantastischen Überformung der "Pedes hypocampi", einem Element der vorderen Hirnhöhle, den Joseph Guichard Duverney im 17. Jahrhundert entdeckt hatte. Die Metaphorik des Begriffs - "Pedes bypocampi" heißt übersetzt: SeepferdchenFüße - wird von der medizinische Kommission verwörtlicht, d.h. als "wohl conservirte[n] Pferdsfüße" (Bogs 45) interpretiert, was es möglich macht, sie als "Ursprung des schwarzen geflügelten Pferdes" (Bogs 46) auszumachen, von dem Bogs schon in seinem Bericht über das Konzert gesprochen hatte.

In dieses "schwarze[ [...] Roß" hatte sich damals der in seinen Fantasien auftauchende "schwarze Engel", also der Teufel oder ein Teufel-ähnliches Wesen, "verwandelt!", als er nicht nur den Kampf um Klarinettens "Seele", die er auf einer "Tonleiter zum Himmel" ziehen lassen musste (Bogs 26f.), sondern auch um Klarinettes Leib verloren hatte, den ihr ehemaliger Liebhaber, Klarin, suspekterweise mittlerweile mit einer Sirene liiert, im Feuer zerstörte hatte.

\footnotetext{
23 Vgl. zur 'Geheimpoetik' des zweiten Teils - insbesondere was das italienische Alter Ego anbetrifft - VillonLechner 1996.
} 
Dass sich dieser Schwarze Engel während der medizinischen Visitation von Bogs' Gehirn als eine "Küchenmagd" (Brentano, S. 46), also als Täuschung der Fantasie, erweist, wurde in der Vision Bogs' während dieses Konzertes vorbereitet. Denn schon damals ähnelte das Pferd, zu dem der schwarze Engel mutiert, einem "geflügeltes Roß" (Bogs 27), also Pegasus oder der dichterischen Fantasie.

Und wo ist der Teufel jetzt? Der Verdacht der Mediziner verlagert sich, entsprechend der verräterischen Schlittenfahrt von Schelmuffsky und Bruder Graf, von der dritten auf die vierte Hirnhöhle. Dieses Ventrikel hat allerdings den entscheidenden Nachteil, dass es - anders als das bisher untersuchte vordere - durch das Sehgerät, das die Riechnerven darstellen, nur sehr schemenhaft einsehbar ist. Die Ärzte "vermutheten" also nur, dass in ihr der "eigentliche] Sitz der Nigromantie" zu finden ist, "denn sie sahen, soviel das Dunkel und das matte Halblicht es erlauben wollten, seltsame Charaktere dort, alle Thierkreiszeichen schienen ihnen lebendig darin umzugehen; Manns- und Weibsvolk, das die sieben Planeten vorstellte, hielten sich in der Grube auf, und sonst viel wunderbares, verdächtiges Gesindel, ein Scheusal mit sieben Köpfen glaubten wir einmal auf einen Blick darunter zu bemerken, aber es ließ sich nichts gewisses darüber entscheiden, weil die Gestalten alle,wie die Figuren durch die Fenster eines Ballsaals auf einen Moment nur sich zeigen wollten, und schnell vorüberschwirrten" (Bogs 47). Wenn man also, so das Räsonnement der Mediziner, des durch Schelmuffsky anzitierten Teufels irgendwie habhaft werden kann, dann in der von Schelmuffsky besuchten vierten Hirnhöhle, deren Uneinsichtigkeit sie doppelt verdächtig macht.

Es stellt sich nun die Frage, was es mit der inspizierten vorderen Hirnhöhle und der uneinsichtigen vierten vom medizinischen Standpunkt aus gesehen auf sich hat. Beginnen wir bei Samuel Thomas Soemmering und seiner gehirnphysiologischen Schrift, dem Organ der Seele (1796), dessen Windungen - den cerebralen wie den argumentativen - die handelnden Ärzte im Bogs gewissenhaft nachgehen. ${ }^{24}$

Auf $A b b .1$, einer Grafik aus dem oben genannten Werk, ist ein senkrechter Durchschnitt des Gehirns zu sehen, oben die vordere Hirnhöhle mit allen ihren Seitenhöhlen, unten die vierte Hirnhöhle. Dass Görres und Brentano genau auf diese beiden Höhlen abstellen, hat einen ganz einfachen Grund: Sie stehen für das Sehen und das Hören, eben jene Sinneserfahrung, die sich in den Visionen Bogs' während des Konzertes affizieren.

Das "Sehenervenpaar【" endet nämlich - wie bei Soemmerring (und auch anderen Gehirnphysiologen) nachzulesen ist - "an den Sehenervenhügeln", welche sich in der "Seitenhirnhöhle", also "am Vordern Ende der Hirnhöhle", befinden, während "die Nerven des Gehörs" in die "Vierte[ Hirnhöhle" münden.

Dieses Faktum ist für Soemmerring von zentraler Wichtigkeit. Denn seine im Organ der Seele eigentlich vorgetragene Entdeckung, dass nämlich die Flüssigkeit der Hirnhöhlen das von der Medizin und Philosophie lang gesuchte Sensorium commune darstellt, birgt die theoretisch nicht zu unterschätzende Gefahr, dass der Mensch zu einem Synästhetiker von Natur aus gemacht

\footnotetext{
${ }^{24}$ Zur Gehirnphysiologie im Bogs, vgl. Welsh 2003, 253ff.; ähnlich dies. 2001; Oehler-Kleins 1990, 270ff. (zum Verhältnis Bogs/Gall), und, die Gehirnphysiologie mit der Monstrologie und der Synästhesie verbindend, Vf. 2007c. Zu Soemmerrings Hirnphysiologie allgemein, vgl. Hagner 1997, 63ff.
} 
wird: Wenn alle Sinnenerfahrung noch einmal in einem Sensorium wiederholt werden, dann ist nicht auszuschließen, dass sie sich in ihm vermischen und wechselseitig affizieren.

Um diese Gefahr nun zu bannen, führt Sommering die größtmögliche Distanz zwischen den beiden Nervenenden ins Feld: Wie die Sehnerven am vorderen, so schließen die Hörnerven am "Hintern Ende" der gesamten Hirnhöhlen-Landschaft an. ${ }^{25}$ Diese räumliche Distanz mache nämlich, so Soemmerrings Rettungsanker, deutlich, dass "einem und demselben Wesen (das ist: der Flüssigkeit der Hirnhöhlen) [...] an verschiedenen Stellen Verschiedenes mitgetheilt" werde, und zwar so, dass es dieses Verschiedene als "Verschiedenes empfindeП" ${ }^{26}$

Die eigentliche Synästhesie-Gefahr, die Soemmering fürchtet, geht jedoch nicht vom Sensorium commune selbst, sondern von seinen Theoretikern aus. Johann Gottfried Herder würde den Ausweg aus der angeblichen Synästhesie-Gefahr, wie ihn Soemmerring vorschlägt, nicht akzeptieren. Herder versteht in Vom Erkennen und Empfinden der menschlichen Seele das "Nervengebäude" als körperliches "Analogon" der "Einbildung" bzw. Einbildungskraft im Körper. Und in dieser, wie er schreibt, "innere[n] Sinnlichkeit" könne es gar nicht anders sein, als dass "Alles zusammenfließe und Eins werde". Die Einbildungskraft bzw. das Nervengebäude bestehen seinen Vorstellungen zufolge "nicht bloß aus Bildern, sondern auch aus Tönen, Worten, Zeichen und Gefühlen, für die oft die Sprache keinen Namen hätte". Und genau von diesem Umstand rührt die Synästhesie her: "Das Gesicht borgt vom Gefühl, und glaubt zu sehen was es nur fühlte. Gesicht und Gehör entziffern einander wechselseitig: der Geruch scheint der Geist des Geschmacks, oder ist ihm wenigstens ein naher Bruder". ${ }^{27}$

Bei Herder, dem Philosophen des Gefühls bzw. Gemeingefühls, übernimmt die Einbildungskraft beziehungsweise das Nervengebäude also nicht nur die Funktion der Synthesis, sondern darüber hinaus die epistemisch und moralisch relevanten Übersetzungsleistungen, die Soemmering dem Verstand allein zusprechen will: Durch die "innerliche] Sinnlichkeit" wird aus "Reiz" und "Empfindung [...] Gedanke [...], Wille, Entwurf, Tat, Handlung: alles durch Einen und denselben Boten". ${ }^{28}$ Dass sich Herder damit einen nervlichen Rückkoppelungseffekt, eben die synästhetische Wahrnehmung, einhandelt, scheint er nicht als Manko, sondern als Zugewinn der inneren Erfahrung zu begreifen.

Zurück zum Synästhetiker Bogs und einer bemerkenswerten Eigenschaft seines Nervensystems. Als die Mediziner zur bereits erwähnten Vivisektion des Gehirns schreiten wollen, lässt sich ihr Patient sehr leicht von einem der anwesenden Ärzte "durch Manipulation in magnetischen Schlaf und Somnambulism versetzen" (Bogs 41) - und zwar in einen sehr tiefen, innerhalb dessen die Mediziner nicht nur via Riechnerven die vordere Hirnhöhle inspizieren, sondern einer von ihnen, der todesmutige Dr. Sphex (eine literarische Verlängerung einer Figur aus Jean Pauls Titan), sogar in die nicht einsehbare vierte Hirnhöhle eindringen kann.

Des kuriosen Uhrmachers hohe Empfänglichkeit für magnetische Manipulationen hängt unmittelbar mit seinem anscheinend außergewöhnlichen Sensorium commune zusammen, das

\footnotetext{
25 Soemmerring: Über das Organ der Seele. In: Soemmerring 1990ff., Bd. IX, 183; 199; 180; 199.

26 Ebd., 199.

${ }^{27}$ Herder: Vom Erkennen und Empfinden. In: Herder 1985ff., Bd. IV, 349-353.

${ }^{28}$ Ebd., 350f. Vgl. hierzu Thiele 1987, 121 ff.; Kimminich 2002, 85ff.
} 
die von Sommering ausgeschlossene, von Herder emphatisch beschworene Synästhesie explizit auszuagieren scheint. Johann Christian Reil, nicht nur der Erfinder der modernen Psychologie, sondern auch einer der führenden Gehirn- und Nervenphysiologen seiner Zeit, ${ }^{29}$ schreibt zum Beispiel über die nervlichen Zustände der Somnambulen: "Im Normalzustande empfindet der Nerve sich nicht selbst, sondern nimmt die Eindrücke bloss an seiner Extremität wahr, und leitet sie dem Gehirn zu; im Somnambulismus wird er leuchtend in allen Punkten, und gleichsam für sich selbst wahrnehmbar". ${ }^{30}$

Das bei den Somnambulen so intensiv arbeitende Gemeingefühl ist also nach Reil eine Form der Selbstbeobachtung der Nerven: Durch dieses Gefühl wird "der Seele der Zustand ihres Körpers vorgestellt [...], und zwar vermittelst der Nerven die allgemein durch den Körper verbreitet sind". ${ }^{31}$ Im Falle einer körperlichen Krankheit, des Wahnsinns oder eben auch des Somnambulismus schaltet der Körper, sozusagen in einem physiologischen Ausnahmezustand, von Fremdwahrnehmung auf Selbstwahrnehmung um. In diesem Falle werden nun aus den ansonsten dunklen und verworrenen Selbstwahrnehmungen klare und deutliche Vorstellungen und damit wird notwendig das ansonsten nur latent wirkende synästhetische Potenzial des gemeinen Gefühls freigesetzt.

Was aber hat das mit der vierten Hirnhöhle zu tun? Es gilt sich in diesem Zusammenhang vor Augen zu führen, dass Brentano und Görres als zerebrales Sensorium commune nicht mehr, wie noch Reil, das Gehirnwasser, sondern den Gehirndunst - in ihren Worten: den "expansible[n] Dunst" - annehmen (genau das hatte nämlich Görres wenige Jahre zuvor in seinen Aphorismen über die Organonomie mit Bezug auf Chladni ${ }^{32}$ behauptet), ${ }^{33}$ was aber an der Sachlage nichts ändert: Auch dieses mobile und flexible allgemeine Sinnenwerkzeug schützt das Gehirn Bogs' im Sinne seiner Erfinder nicht vor Überlagerung der verschiedener Nerven-Übertragungen, sondern macht vielmehr die synästhetische Verbindung überhaupt erst möglich.

So steht es auch im Visum repertum: Am Beginn der, zu diesem Zeitpunkt noch visuellen, Expedition in Bogs' Gehin werden die beteiligten Mediziner mit einem Phänomen in der vorderen Hirnhöhle konfrontiert, das von ihnen als der "böse Schwaden, der Gehirndunst" (Bogs 42) bezeichnet wird. Recht bald wird deutlich, dass dieser Schwaden aus der vierten Hirnhöhle kommt: Die Luft ist, wie der abgeseilte Dr. Sphex seinen Kollegen wenig später von unten berichten kann, hier geradezu "mephitisch", was auch die zurückgebliebenen Mediziner zu spüren bekommen: Nach einer verunglückten Reinigungsaktion stürzt "ein Schwall von garstigen, mephitischen Luftarten" aus der "Öffnung" der vierten Hirnhöhle hervor (Bogs 48f.).

Das könnte der Grund sein, warum die vierte Hirnhöhle von den Medizinern so verteufelt wird: Sie ist die Ursache der synästhetischen Wahrnehmungen Bogs'. Aus ihr als des Teufels eigentlicher Küche dringt der Gehirndunst - also das wahre Sensorium commune oder das

\footnotetext{
${ }^{29}$ Vgl. hierzu Hagner 1997, $157 \mathrm{ff}$.

${ }^{30}$ Reil 1807, 233f.

31 Reil 1817, 38; 77; 108.

32 Chladni 1802, 285, kann nicht verstehen, "warum ein" in den Hirnhöhlen "enthaltener feuchter Dunst, oder eine gasförmige Flüssigkeit nicht sollte eben die Geschäfte verrrichten können, die dort einer wäßrigen Flüssigkeit zugeschrieben werden". Vgl. hierzu Welsh 2003, $272 \mathrm{f}$.

${ }^{33}$ Görres: Aphorismen über die Organonomie. In: Görres 1926ff., Bd. II.1, 190.
} 
Gemeingefühl - und überträgt die dort empfangenen Nerven-Informationen, das heißt die auditiven Wahrnehmungen, in den Rest des Gehirns, d. h. in die vordere Hirnhöhle. Da die anderen (insbesondere die Seh-)Nerven zur Zeit des Konzertes weitgehend arbeitslos sind und Bogs über ein pathologisch sensibilisiertes Gemeingefühl verfügt, nehmen diese die auditiven, durch den Dunst übertragenen, Informationen auf ihre Weise, also bildlich, wahr. Voilà die perfekte gehirnphysiologische Rekonstruktion einer synästhetischen Wahrnehmung (die die kleinen Soemmerringe in Bogs' Gehirn eben nur als teuflisch wahrnehmen können).

\section{Was hat der schelmuffskysche Teufel in der vierten Hirnböble zu suchen?}

Halten wir also fest: Die vierte Hirnhöhle steht, gehirnphysiologisch betrachtet, nicht nur für den Hörsinn, sondern - da die durch den Gehirndunst übertragenen informationsschwachen auditiven Sinnesdaten zu einer visuellen Überlagerung geradezu aufrufen - für die Fähigkeit zur synästhetischen Wahrnehmung. Ganz im Gegenteil zur vorderen Hirnhöhle, die nicht nur für die Gesichtswahrnehmung allein verantwortlich zeichnet, sondern für die ursprüngliche Distinguierung dieser Sinnesdaten von allen anderen.

Was hat das nun aber mit dem über den Schelmuffsky anzitierten Teufel zu tun, der ja, wie gesagt, in dieser vierten Hirnhöhle zu logieren scheint? Ich habe oben auszuführen versucht, dass Reuters Erzähler seinen literarischen Vater nur noch schemenhaft an der Textoberfläche thematisiert, weil er stattdessen das von ihm erhaltene Erbgut zur Textproduktion verwendet, also selbst mit seiner Erzählung die teuflische Technik, via "Fantasey" den Menschen "einen blauen Dunst für die Augen [zu] mache[n]", ausübt. ${ }^{34}$

Und das gilt für den Bogs auch. Für Herder ist nämlich, wie oben ausgeführt, das Sensorium commune das nervliche Analogon zur Einbildungskraft und damit, zumindest wenn man ihren produktiven Teil betrachtet, zur Fantasie. So sieht es auch der Herder-Leser Jean Paul: "Die fünf Sinne [...] drücken die Natur mit fünf verschiedenen Platten ab", die "Phantasie" hingegen "als sensorium commune liefert sie alle mit einer". ${ }^{35}$ Von dieser Perspektive aus gesehen wird deutlich, warum der durch den Schelmuffsky anzitierte Teufel in der (für die Synästhesie verantwortlichen) vierten Hirnhöhle von Bogs Residenz bezogen hat: Hier hat seine Fähigkeit, den Menschen Fantasien anstelle der realen Wahrnehmung zu implementieren, seinen ursprünglichen Ort. Hier kommt der Teufel, so könnte man sagen, gehirnphysiologisch zu sich selbst.

Und da er es sich in der vierten Hirnhöhle nun schon gemütlich gemacht hat, kann der literarische Teufel seine zweite oben geschilderte Eigenschaft, nämlich die zur parodischen Verzerrung vorgegebenen Materials zum Zwecke eines karnevalesken Lachens, gleich mit zur Anwendung bringen. Denn wenn man - wie im Bogs geschehen - Fantasie über Synästhesie denkt, dann spricht man ihr nicht die Fähigkeit zur Fiktion, sondern lediglich zur Neuorganisation von tatsächlicher Sinneswahrnehmung bzw. Nerveninformation zu. Und genau das, so möchte ich argumentieren, gilt für die nach außen, also in die Texte, gekehrte Fantasie der Parodie ebenfalls: Auch sie erfindet nicht neu, sondern arrangiert das bestehende Material so, dass es in das Gegenteil der ursprünglichen textuellen Intention umschlägt. Das ist der oben erwähnten teuflische "Zusatz", der die nachgeäffte Vorgabe in ihr Gegenteil verkehrt.

\footnotetext{
${ }^{34}$ Harsdörffer 1974, 434.

35 Jean Paul: Über die natürliche Magie der Einbildungskraft. In: Jean Paul 1959-1985, Bd. I.4, 195.
} 
Zu dieser parodischen Technik gehört die, im zeitgenössischen Diskurs topische Behauptung, dass man das teuflische Element den Vorgängertexten nicht nachträglich zugefügt, sondern es in ihnen entdeckt und lediglich freigelegt habe. ${ }^{36}$ Dies gilt für Schelmuffky wie Bogs gleichermaßen: Wie Schelmuffsky seine äußerlichen Reisen, ich habe das oben am Beispiel des Besuchs beim Großmogul ausgeführt, als Lügengeschichte ausstellt und damit die bestehende Reisliteratur durch synästhetische oder eben parodische Umorganisation eben dessen beschuldigt, so sind auch die Reisen in Bogs Gehirn eine Lügengeschichte über Lügengeschichten. Schließlich kann sich Bogs - aller Vivisektion und cerebralen Zweiteilung wunderbar unbeschadet - am Ende als Generalautor seiner in den "badische[n] Wochenschrift" (Bogs 52) abgedruckten Geschichte präsentieren. Er macht durch diesen Hinweis deutlich, dass er mithilfe von Schelmuffskys Lügenund Fantasiegeschichte auch das Fantastische der zeitgenössischen Gehirnphysiologie parodisch offenzulegen in der Lage ist.

Alle äußerlichen und innerlichen Reiseberichte, so seriös und wissenschaftliche sie sich immer geben, bergen also, so lassen Schelmuffsky und Bogs ihre Leser augenzwinkernd wissen, einen fantastischen oder eben teuflischen Kern. Und eben diesen Kern legen die beiden Erzähler in ihren Parodien dieser Texte als den eigentlichen Motor der Erzählung bloß. Mit einer solchen komischen Geste der Inversion werden die beiden Erzähler - im Gegensatz zu den von ihnen parodierten Texten - dem Prinzip des literarischen Teufels erst wirklich gerecht, denn dieser ist, wie es in Jean Pauls Grönländischen Prozessen heißt, "der Vater der Lügen aber blos aus Humor". ${ }^{37}$

\section{Bibliografie}

Bachtin M M (1979) Die Ästhetik des Wortes. Übers. von Grübel R, Reese S. Frankfurt: Suhrkamp

Bachtin M M (1995) Rabelais und seine Welt. Volkskultur als Gegenkultur. Übers. von Leupold G. Frankfurt a. M.: Suhrkamp

Bauer M (1994) Der Schelmenroman. Stuttgart: Metzler

Bergengruen M (2003) Schöne Seelen, groteske Körper. Jean Pauls ästhetische Dynamisierung der Anthropologie. Hamburg: Meiner

Bergengruen M (2005) Die heitere Therapie. Persönlichkeitsspaltung und Groteske in E.T.A. Hoffmanns 'Prinzessin Brambilla'. In: Colloquium helveticum 20: 119-142

Bergengruen M (2007a) Nachfolge Christi - Nachahmung der Natur. Himmlische und natürliche Magie bei Paracelsus, im Paracelsismus und in der Barockliteratur (Scheffler, Zesen, Grimmelshausen). Hamburg: Meiner

Bergengruen M (2007b) Der Große Mogol oder der Vater der Lügen des Schelmuffsky. Zur Parodie des Reiseberichts und zur Poetik des Diabolischen bei Christian Reuter". In: Zeitschrift für Deutsche Philologie 126

Bergengruen M (2007c) Das Geheimnis der vierten Hirnhöhle. Zum Zusammenhang von Physiologie, Monstrologie und Synästhesie in Brentanos und Görres' 'Bogs'. Erscheint in: Landfester U, Simon R (Hrsg.) Clemens Brentano. Poesie der Zirkulation, Würzburg: Königshausen und Neumann

\footnotetext{
${ }^{36}$ Vgl. zu dieser parodischen Strategie, insbesondere bei Jean Paul, Vf. 2003, $200 \mathrm{ff.}$

37 Jean Paul 1959-1985, Bd. II.2, 189 f.
} 
Brentano C / Görres J (anon.) (1807) Entweder wunderbare Geschichte von Bogs dem Uhrmacher, wie er zwar das menschliche Leben längst verlassen, nun aber doch, nach vielen musikalischen Leiden zu Wasser und zu Lande, in die bürgerliche Schützengesellschaft aufgenommen zu werden Hoffnung hat, oder die über die Ufer der Badischen Wochenschrift als Beilage ausgetretene Konzert-Anzeige. Nebst des Herrn Bogs wohlgetroffenem Bildnisse und einem medizinischen Gutachten über dessen Gehirnzustand. Heidelberg: Mohr \& Zimmer (Sigle 'Bogs')

Chladni E F F (1802) Die Akustik, Leipzig: Breitkopf und Härtel

Deneke O (1927) Schelmuffsky. Ein Vortrag, Göttingen: Göttingische Nebenstunden

Elsaghe Y A (1991) Schelmuffsky als närrischer Odysseus. In: Simpliciana 13: 485-492

Fechner J-U (1982) Schelmuffskys Maskeraden und Metamorphosen. Neue Forschungsaspekte zu Christian Reuter. In: Euphorion 76: 1-26

Geulen H (1972) Noten zu Christian Reuters 'Schelmuffsky'. In: Rasch W et al. (Hrsg.) Rezeption und Produktion zwischen 1570 und 1730. FS für Günther Weydt. Bern, München: Francke, 481-492

Görres J (1926ff.) Gesammelte Schriften. Hrsg. von Schellberg W et al. Paderborn et al.: Schöningh et al.

Herder J G (1985ff.) Werke in zehn Bänden. Hrsg. von Bollacher M et al. Frankfurt a. M.: Bibliothek deutscher Klassiker

Grimm G E (1992) Christian Reuter: 'Schelmuffskys warhafftige curiöse und sehr gefährliche Reisebeschreibung zu Wasser und Lande'. Kapriolen eines Taugenichts. Zur Funktion des Pikarischen. In: Baeumer M L (Hrsg.) Interpretationen. Romane des 17. und 18. Jahrhunderts. Stuttgart: Reclam, 47-75

Grimmelshausen H J C v. (1989-1997) Werke. Hrsg. von Breuer D. 3 Bde. Frankfurt a. M.: Bibliothek deutscher Klassiker

Hagner M (1997) Homo cerebralis. Der Wandel vom Seelenorgan zum Gehirn, Berlin: Berlin Verl.

Harsdörffer G P (1974) Der Grosse Schau-Platz jämmerlicher Mord-Geschichte. Hildesheim, New York: Olms (=ND der Ausgabe Hamburg 1656)

Hecht W (1966) Christian Reuter. Stuttgart: Metzler

Jean Paul (1959-1985) Werke. Hrsg. von Miller N et al., 10 Bde., München: Hanser

Kaminski N (2004) Von Plißine nach Schelmerode. Schwellenexperimente mit der 'Frau Mutter Sprache' in Christian Reuters 'Schlampampe'-Projekt. In: Heudecker S et al. (Hrsg.) Kulturelle Orientierung um 1700. Traditionen, Programme, konzeptionelle Vielfalt, Tübingen: Niemeyer, 236-262

Kimminich E (2002) Synästhesie und Entkörperung der Wahrnehmung. Bemerkungen zu einer historischen Entwicklung in Europa vom 17. bis zum 20. Jahrhundert. In: Semiotik 24.1: 71 109

König H (1945) Christian Reuters 'Schelmuffsky' als Typ der barocken Bramarbas-Dichtung. Hamburg 1945: masch. Diss.

Lubkoll C (1995) Mythos Musik. Poetische Entwürfe des Musikalischen in der Literatur um 1800. Freiburg: Rombach

Luther M (1883ff.) Werke. Kritische Gesamtausgabe (Weimarer Ausgabe). Weimar et al.: Böhlau 
Moscherosch H M (1964) Gesichte Philanders von Sittewald. Hrsg. von Bobertag F. Darmstadt: WBG (=ND der Ausgabe Stuttgart 1883)

Müller K-D (1994) Einfallslosigkeit als Erzählprinzip. Zu Christian Reuters 'Schelmuffsky'. In: Esselborn H, Keller W (Hrsg.) Geschichtlichkeit und Gegenwart. FS für Hans Dietrich Irmscher. Köln et al.: Böhlau, 1-12

Oehler-Kleins S (1990) Die Schädellehre Franz Joseph Galls in Literatur und Kritik des 19. Jahrhunderts, Stuttgart, New York: G. Fischer

Reil J C (1807) Ueber die Eigenschaften des Ganglien-Systems und sein Verhältnis zum CerebralSystem. In: Archiv für die Physiologie 7: 189-254

Reil J C (1817) Ueber das Gemeingefühl. In: ders.: Kleine Schriften wissenschaftlichen und gemeinnützigen Inhalts, Halle: Curt, $34-112$

Reuter C (anon.) (1696), Schelmuffskys Warhafftige Curiöse und sehr gefährliche Reisebeschreibung zu Wasser und Lande. I. Theil / Und zwar die allervollkommenste und accurateste Edition in Hochteutscher Frau Mutter Sprache eigenhändig und sehr artig an den Tag gegeben von E. S. "Schelmerode" (T.1, B-Auflage)

Reuter C (anon.) (1697), Schelmuffskys curiöser und sehr gefährlicher Reise-Beschreibung Zu Wasser und Lande Anderer Theil. "Padua" (T. 2, A-Auflage)

Reuter C (anon.) (1750), Schelmuffskys wahrhaftige, curiöse und sehr gefährliche ReiseBeschreibung zu Wasser und Lande in zweyen Theilen [...], Frankfurt, Leipzig (Sigle 'Schelmuffsky')

Reuter C (21956) Schelmuffsky. Hrsg. von Polenz P v. Halle (Saale): Niemeyer

Reuter C (1997), Schelmuffskys warhafftige curiöse und sehr gefährliche Reisebeschreibung zu Wasser und Lande. Hrsg. von Barth I-M. Stuttgart: Reclam

Rölleke H (2004) Clemens Brentanos Entwurf eines Gänsespielbretts nach Christian Reuters barockem 'Schelmuffsky'-Roman. In: Jahrbuch des Freien Deutschen Hochstiftes 44: 197-233

Rölleke H (2005) Christian Reuters Barockroman 'Schelmuffsky' und die 'Kinder- und Hausmärchen der Brüder Grimm. In: Fabula 46: 305-308

Tatlock L (1992) Quixotic marvel. Emesis and the miscarriage of subjektivity in Christian Reuter's 'Schelmuffsky'. In: Hardin J et al. (Hrsg.) 'Der Buchstab tödt - der Geist macht lebendig'. FS für Hans-Gert Roloff. 2 Bde. Bern et al.: Lang, Bd. I, 297-319

Thiele B F M (1987) Herders Theorie des synästhetischen Wahrnehmens zur Grundlegung der ästhetischen Erziehung, Tübingen: masch. Diss.

Soemmerring S T (1990ff.) Werke. Hrsg. von Mann G, Benedum J et al., Basel: Schwabe, Bd. IX

Utz P (1990) Das Auge und das Ohr im Text. Literarische Sinneswahrnehmung in der Goethezeit. München: Fink

Villon-Lechner A (1996) Der entschwundene Erzähler. Zur Selbstreflexion des Mediums in Christian Reuters Roman 'Schelmuffsky'. In: Simpliciana 8: 89-96.

Welsh C (2001) Wie aus Tönen Bilder werden. Zur Figuration der Musik und ihrer Kritik. In: dies. et al. (Hrsg.) Sinne und Verstand. Ästhetische Modellierungen der Wahrnehmung um 1800. Würzburg: Königshausen und Neumann, 169-188

Welsh C (2003) Hirnhöhlenpoetiken. Theorien zur Wahrnehmung in Wissenschaft, Ästhetik und Literatur um 1800. Freiburg: Rombach 
Weyer J (1967) De praestigiis demonvm. Von ihrem vrsprung / vnderscheid / vermögenheit / vnd rechtmeßiger straaff / auch der beleidigten ordenlicher hilff / sechs Bücher. Übers. von dems. Amsterdam: Bonset (=ND der Ausgabe 1578)

Weyer J (1586) De praestigiis daemonvm. Von Teuffelsgespenst Zaubereren vnd Gifftbereytern / Schwartzkünstlern / Hexen vnd Vnholden [...]. Übers. von Fuglinus J. Frankfurt a. M.: Basseus 